\title{
Intratumoral androgen levels are linked to TMPRSS2-ERG fusion in prostate cancer
}

\author{
Matias Knuuttila1,2, Arfa Mehmood3, Jenni Mäki-Jouppila1,2, Henrik Ryberg4, Pekka Taimen5, Juha Knaapila6, \\ Otto Ettala6, Peter J Boström6, Claes Ohlsson4, Mikko S Venäläinen³, Asta Laiho3, Laura L Elo3, Petra Sipilä1,2, \\ Sari I Mäkelä2,7 and Matti Poutanen1,2,4
}

${ }^{1}$ Research Centre for Integrative Physiology and Pharmacology, Institute of Biomedicine, University of Turku, Turku, Finland

${ }^{2}$ Turku Center for Disease Modeling (TCDM), Institute of Biomedicine, University of Turku, Turku, Finland

3Turku Centre for Biotechnology, University of Turku and Åbo Akademi University, Turku, Finland

${ }^{4}$ Center for Bone and Arthitis Research, The Sahlgrenska Academy, University of Gothenburg, Gothenburg, Sweden

${ }^{5}$ Department of Pathology, University of Turku and Turku University Hospital, Turku, Finland

${ }^{6}$ Department of Urology, Turku University Hospital, Turku, Finland

7Functional Foods Forum, University of Turku, Turku, Finland

Correspondence should be addressed to M Poutanen: matti.poutanen@utu.fi

\begin{abstract}
Intratumoral androgen biosynthesis is one of the mechanisms involved in the progression of prostate cancer, and an important target for novel prostate cancer therapies. Using gas chromatography-tandem mass spectrometry and genome-wide RNA sequencing, we have analyzed androgen concentrations and androgen-regulated gene expression in cancerous and morphologically benign prostate tissue specimens and serum samples obtained from 48 primary prostate cancer patients. Intratumoral dihydrotestosterone (DHT) concentrations were significantly higher in the cancerous tissues compared to benign prostate $(P<0.001)$. The tissue/serum ratios of androgens were highly variable between the patients, indicating individual patterns of androgen metabolism and/or uptake of androgens within the prostate tissue. An unsupervised hierarchical clustering analysis of intratissue androgen concentrations indicated that transmembrane protease, serine 2/ETS-related gene (TMPRSS2-ERG)-positive patients have different androgen profiles compared to TMPRSS2ERG-negative patients. TMPRSS2-ERG gene fusion status was also associated with an enhanced androgen-regulated gene expression, along with altered intratumoral androgen metabolism, demonstrated by reduced testosterone concentrations and increased DHT/ testosterone ratios in TMPRSS2-ERG-positive tumors. TMPRSS2-ERG-positive and -negative prostate cancer specimens have distinct intratumoral androgen profiles, possibly due to activation of testosterone-independent DHT biosynthesis via the alternative pathway in TMPRSS2-ERG-positive tumors. Thus, patients with TMPRSS2-ERG-positive prostate cancer may benefit from novel inhibitors targeting the alternative DHT biosynthesis.
\end{abstract}
Key Words
- intratumoral
- androgen
- prostate
- cancer
TMPRSS2-ERG

Endocrine-Related Cancer (2018) 25, 807-819

\section{Introduction}

Androgens play a crucial role in the normal development and growth of the prostate and the growth of prostate cancer. The prostate tissue is known for the local biosynthesis of dihydrotestosterone (DHT), which is the principal androgen in the prostate, produced from the circulating testosterone via the activity of $5 \alpha$-reductases 
(Wilson 2001). However, recent studies have demonstrated that DHT is also synthesized independent of testosterone by alternative pathways, particularly in castrationresistant prostate cancer (CRPC) (Chang et al. 2011).

Intratumoral androgen biosynthesis, either from the adrenal precursors or by de novo synthesis from cholesterol, has been established to be one of the mechanisms involved in the progression of prostate cancer to castration resistance (Mostaghel \& Nelson 2008, Cai \& Balk 2011). While androgen deprivation therapy (ADT) efficiently reduces the level of circulating testosterone, high concentrations of testosterone and DHT persist in the prostate tissue (Titus et al. 2005, Page et al. 2006). Accordingly, CRPC remains an androgen-regulated disease, and the treatments inhibiting intratumoral androgen biosynthesis have proven to be effective for treating CRPC patients (de Bono et al. 2011, Ryan et al. 2013). The genome-wide transcriptome analyses of CRPC specimens have provided evidence for the presence of a large number of enzymes belonging to the short-chain dehydrogenase/reductase, aldo-keto reductase and CYP families, many of them potentially being involved in androgen biosynthesis and metabolism (Penning 2014). Moreover, new enzymes playing a role in the nonclassical pathways for the production of active androgens in prostate cancer have been identified (Mostaghel 2014, Zhang et al. 2016).

Although the role of intratumoral androgen biosynthesis in CRPC is well established, its presence in primary prostate cancer has not been extensively studied. The present published data of intraprostatic androgen concentrations is largely inconsistent, due to the use of non-specific immunoassays and the limited number of specimens (Van Der Sluis et al. 2012). So far, no clear differences in androgen concentrations in the normal, benign hyperplastic and cancerous prostate tissues have been observed (Van Der Sluis et al. 2012). Furthermore, it is not known if/how serum androgen levels reflect intraprostatic androgen levels in treatment-naïve prostate cancer patients.

High-performance liquid chromatography-tandem mass spectrometry and gas chromatography-tandem mass spectrometry (GC-MS/MS)-based methods, developed by us and others (Keski-Rahkonen et al. 2011, Tamae et al. 2013, Nilsson et al. 2015, Quanson et al. 2016), make it possible to reliably detect multiple steroids simultaneously in body fluids and tissue samples. We have demonstrated the usefulness of these assays in the analysis of both clinical specimens and preclinical models (Huhtinen et al. 2014, Knuuttila et al. 2014). In the present study, we analyzed the androgen concentrations in cancerous and morphologically benign prostate tissue samples and serum of 48 patients, who underwent radical prostatectomy due to localized prostate cancer. Our objective was to characterize putative cancer-related changes in intratissue androgen profiles, including associations with the expression of AR target genes. Understanding the pathways of intratumoral androgen biosynthesis in primary prostate cancer may lead to identification of novel drug targets and pave the way to new personalized therapeutic strategies.

\section{Materials and methods}

\section{Patients}

Prostate samples were collected from patients diagnosed with localized adenocarcinoma of prostate. The patients underwent robot-assisted laparoscopic radical prostatectomy (RALP) at Turku University Hospital between the years 2013 and 2015. Extended pelvic lymph node dissection was performed in high-risk cases (biopsy Gleason $\geq 4+3$, prostate-specific antigen (PSA) $\geq 15 \mathrm{ng} / \mathrm{mL}$ or clinically advanced $\mathrm{T} 3$ tumor). The study protocol was approved by the Ethics Committee of the Hospital District of Southwest Finland. A written consent was obtained from all patients participating to the study conducted according to the principles in the Declaration of Helsinki. The following inclusion criteria were applied to select patients to the study cohort: diagnosis of localized adenocarcinoma of prostate, pre-operative and postoperative Gleason score $\geq 6$ and detectable pre-operative serum PSA level. The representative cancerous and benign samples with good RNA integrity were required from each patient, limiting the number of patients that were eligible for the study cohort $(n=48)$. The clinicopathological characteristics of the patients, such as pre- and post-operative PSA values, stage (pTNM), lymph node metastasis, disease recurrence (biochemical and clinical recurrence) and history of hormonal treatments were collected from the Turku University Hospital records by an experienced urologist and are shown in Table 1 . The PSA threshold for biochemical recurrence was set at $0.2 \mathrm{ng} / \mathrm{mL}$ and the follow-up period was $>2$ years post radical prostatectomy.

\section{Sample collection}

All sample collection procedures took place in the operation room during RALP operation. Serum samples 
Table 1 Clinicopathological characteristics of study cohort.

Number of patients
Age
Pre-operational PSA (ng/mL)
Prostate weight (g)
Gleason score
6
7
8
9
10
pT category
pT2
pT3a
pT3b
pT4
pN category (lymph node)
Positive (Npos)
Negative (Nneg)
No lymphadenectomy (Nx)
pM category (metastasis)
Positive (M1)
Negative (M0)
Surgical margin
Positive
Negative
Hormonal medication
No hormonal therapies
5 -alfa reductase inhibitor (dutasteride)
5 -alfa reductase inhibitor (finasteride)
GnRH antagonist (Degarelix)

were collected at the time of anesthesia induction, and prostate tissue was processed immediately after surgery. The fresh tissue specimens were processed within $30 \mathrm{~min}$ post-operatively with the help of Auria Biobank (Turku), using the following standard biobanking procedure: after staining the surgical margins with tissue ink, the most apical and basal regions of the prostate were removed. From the remaining tissue, representing approximately $80 \%$ of the whole prostate, three tissue cores were removed (right posterior, left posterior and anterior regions of the prostate) in apical-basal axis using a MD5000 Tissue Coring Press (Alabama Research \& Development, Munford, USA) with coring tools ranging in size from 5 to $8 \mathrm{~mm}$. Each core, ranging between 20 and $40 \mathrm{~mm}$ in length (depending on the size of the prostate), was split into three parts and a histological validation sample was cut from the middle of each tissue piece yielding in total nine samples/core. The validation samples were fixed in $10 \%$ formalin, embedded in paraffin and stained with hematoxylin and eosin after routine histopathological process. The fresh tissue material next to validation samples was frozen and stored for further analyses.

\begin{tabular}{|c|c|}
\hline Median & Min, $\max$ \\
\hline 48 & \\
\hline 65 & 52,72 \\
\hline 9.9 & $3.4,61.0$ \\
\hline 50 & 22,80 \\
\hline Number of samples & (\%) \\
\hline 1 & 2.1 \\
\hline 33 & 68.8 \\
\hline 1 & 2.1 \\
\hline 11 & 22.9 \\
\hline 2 & 4.2 \\
\hline 13 & 27.1 \\
\hline 21 & 43.7 \\
\hline 12 & 25.0 \\
\hline 2 & 4.2 \\
\hline 10 & 20.8 \\
\hline 26 & 54.2 \\
\hline 12 & 25 \\
\hline 0 & 0 \\
\hline 48 & 100 \\
\hline 21 & 43.7 \\
\hline 27 & 56.3 \\
\hline 38 & 79.2 \\
\hline 6 & 12.5 \\
\hline 3 & 6.3 \\
\hline 1 & 2.1 \\
\hline
\end{tabular}

In the histological analysis of validation samples, the percentage of stroma, normal epithelium, prostatic intraepithelial lesion (PIN) and carcinoma, as well as Gleason grade and presence of inflammation (score 0-3) were determined for each tissue sample. For steroid and RNA-sequencing analysis, the fresh frozen samples next to validation samples containing minimum 50\% of Gleason score $\geq 6$ carcinoma (tumor sample) as well as morphologically benign prostatic tissue (benign control sample) from the same patient $(n=48)$ were selected. The scheme for the sample collection and histological validation is described in Supplementary Fig. 1 (see section on supplementary data given at the end of this article). Serum samples for steroid analysis were collected from the patients at the time of prostatectomy, aliquoted and stored at $-80^{\circ} \mathrm{C}$ until used.

\section{Measurement of serum and intratissue steroids and serum sex hormone-binding globulin}

Tissue samples (weight varying 15-50 mg) for steroid

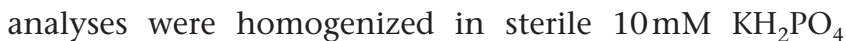


using an Ultra Turrax homogenizer (IKA-Werke, Staufen im Breisgau, Germany). After homogenization, the samples were centrifuged at $800 \mathrm{~g}$ for $10 \mathrm{~min}$ at $4^{\circ} \mathrm{C}$ and the supernatant was collected. The remaining cell pellet, with a small amount of supernatant $(\sim 100 \mu \mathrm{L})$, was further homogenized for $1 \mathrm{~min}$ using a Tissuelyzer LT homogenizer (Qiagen) using a steal bead with a diameter of $5 \mathrm{~mm}$. The homogenate was combined with the supernatant collected in the first step of the homogenization, and the samples were stored at $-80^{\circ} \mathrm{C}$. The steroid analyses of tissue and serum samples were conducted using a GC-MS/MS according to a method previously described (Nilsson et al. 2015). To compare tissue and serum steroid concentrations, $1 \mathrm{~g}$ of tissue sample was considered to be equivalent to $1 \mathrm{~mL}$ of serum. The lower limits of quantitation for DHEA, androstenedione (A-dione), testosterone and DHT were $50,6,8$ and $3.75 \mathrm{pg} / \mathrm{mL}$, respectively. Sex hormonebinding globulin levels of serum samples were measured using ECL-based immunoassay with a Cobas E 602 modular analyzer (Roche Diagnostics) at Turku University Hospital Laboratory. The amount of free testosterone in serum was calculated using a previously published method (Vermeulen et al. 1999). The total androgenicity index was calculated as the sum of $(1 \times \mathrm{T})+(5 \times \mathrm{DHT})$ (Mostaghel 2014).

\section{RNA extraction and RNA sequencing}

For RNA analyses, tissue samples were homogenized using an Ultra Turrax homogenizer. Total RNA for RNA sequencing (RNA-seq) was extracted using NucleoSpin RNA kit and NucleoSpin RNA/DNA buffer set (MachereyNagel, Düren, Germany) according to manufacturer's instructions. The RNA quality was confirmed using a Fragment Analyzer (Advanced Analytical Technologies, Ankeny, IA, USA), and RNA quality number of all the samples used in the study was $\geq 5.5$. The RNA-seq analyses of cancerous $(n=48)$ and benign $(n=48)$ tissue samples were carried out at the Finnish Functional Genomics Center, Turku, Finland.

FastQC (v0.11.3) tool was used to examine the quality of the sequenced RNA-seq data. The reads were aligned to the human reference genome (version hg19) available at UCSC (downloaded from Illumina iGenome website) using Star software v2.5.0c. The alignment bam files from different lanes were merged using Picard tools (v1.77). The Subread tool v1.5.0 was used to count the uniquely mapped reads associated with each gene using Refseq annotation.

\section{Immunohistochemistry}

To confirm the findings of RNA-seq analyses, the transmembrane protease, serine 2/ETS-related gene (TMPRSS2-ERG) fusion status in five tumors was studied with immunohistochemistry (IHC). In brief, sections of 3-4 $\mu \mathrm{m}$ in thickness from original validation samples were cut, the paraffin was removed with xylene and the sections were rehydrated with series of ethanol. The heat-induced antigen retrieval was performed in a microwave oven using Target Retrieval Solution (Dako) followed by cooling at RT. The sections were incubated for 1 hour at RT in a humid chamber with rabbit monoclonal ERG antibody (1:250; clone EPR3864; Epitomics). The primary antibody was detected with EnVision + Dual Link System-HRP (Dako) goat-anti-rabbit secondary antibody for $30 \mathrm{~min}$ at RT. The staining was visualized with $\mathrm{DAB}+$ chromogen solution (Dako) for $10 \mathrm{~min}$ at RT. After counterstaining with hematoxylin, dehydration and treatment by xylene, the slides were analyzed by an experienced uropathologist (P T). The vascular endothelial cells served as positive controls for the staining with ERG antibody and cells of the benign glands as the negative control.

\section{Statistical analyses}

GraphPad Prism 7.02 software (GraphPad Software) and $\mathrm{R}$ version 3.2.2 were applied for basic statistical analyses. Wilcoxon matched-pairs signed-rank test and MannWhitney $U$ test were used for comparing the androgen levels between the cancerous, benign and serum samples of the patients. Two-sample $t$-test was used for analyzing androgen levels and RNA-seq gene expression on a single gene level between TMPRSS2-ERG-positive and TMPRSS2ERG-negative samples. The correlation of ERG and CRISP3 gene expression in cancer samples was performed using Pearson correlation. The $P$ values in the basic statistical analyses were not adjusted for multiple testing.

The downstream analysis of the gene counts was performed using $\mathrm{R}$, version 3.2.2 and Bioconductor, version 2.14. The counts were normalized for library size using the Trimmed Mean of M-values (TMM) approach in the R/Bioconductor package edgeR (Robinson et al. 2010). The normalized counts were transformed using the voom approach in the Limma R/Bioconductor package (Ritchie et al. 2015). The differentially expressed genes between TMPRSS2-ERG-positive and TMPRSS2-ERG-negative samples were tested using the Reproducibility-Optimized Test Statistics (ROTS), which optimizes the reproducibility 
among a family of modified t-statistics and is available in the R/Bioconductor package ROTS (Seyednasrollah et al. 2015). FusionCatcher v0.99.6a (Nicorici et al. 2014) along with the associated ENSEMBL database automatically downloaded by the program was used to find the fusion genes in the samples. Hierarchical clustering of the steroid profiles, studied as log2-transformed cancer/ benign ratios of DHEA, A-dione, testosterone and DHT and the normalized expression values of the differentially expressed AR target genes were performed using Euclidean distance and the Ward's method implemented in the $\mathrm{R}$ package pheatmap (https://CRAN.R-project. org/package=pheatmap). The differences in the first occurrence of biochemical recurrence between the clusters were studied using the Kaplan-Meier estimates of survival implemented in the R package survival (https://CRAN.Rproject.org/package=survival).

\section{Results}

\section{DHT levels are higher in cancerous prostate specimens compared to benign prostate}

For intratissue steroid analyses, we selected 48 patients, from whom well representative cancer and benign tissue samples were available and compared the intratissue concentrations of DHEA, A-dione, testosterone and DHT. On an average, DHT concentrations were significantly higher in the cancerous tissue compared to benign prostate in hormonal treatment-naïve patients (median concentrations $3012 \mathrm{pg} / \mathrm{g}$ and $2264 \mathrm{pg} / \mathrm{g}$, respectively, $P<0.001$, Table 2), while no statistically significant differences were observed in DHEA, A-dione and testosterone (Table 2). Moreover, the total androgenicity index was higher in the cancerous tissue compared to benign prostate in hormonal treatment-naïve patients $(P<0.001$, Table 2$)$. Of all the steroids measured, DHEA represented the highest intratissue concentrations in both cancer and benign tissues, being a precursor for local production of active androgens. As expected, intratissue androgen concentrations were significantly different in patients who had received hormonal therapies compared to hormonal treatment-naïve patients. The median intratissue DHT concentrations were 3-fold lower in cancer tissue $(P<0.001)$ and in benign tissue $(P=0.0062)$ in the patients treated with $5 \alpha$-reductase inhibitors when compared to those who did not receive medication (Table 2). The low DHT concentrations were accompanied with significantly elevated testosterone concentrations in cancerous $(P<0.001)$ and benign samples $(P=0.016)$, while the concentrations of DHEA were not statistically significantly altered (cancer: $P=0.804$; benign: $P=0.665$ ). One patient, who had received ADT prior to prostatectomy, had expectedly low, yet still measurable concentrations of testosterone and DHT in serum, and the concentration in tumor was 50-fold lower compared to the median concentration in the men, who were not treated with any hormonal therapies (Table 2). Interestingly, the total androgenicity indexes in the cancerous samples from the ADT-treated patient and SRD5A-inhibitor-treated patients were very similar (Table 2). These results confirmed the accuracy of our GC-MS/MS method for measuring significant changes in the androgen concentrations of tissue and serum samples obtained from prostate cancer patients.

\section{Intratissue androgen profiles in cancerous and benign samples are highly individual among prostate cancer patients}

Tissue/serum ratios of the steroids measured were calculated for each patient, and DHT concentrations in the cancerous and benign tissues were found 10-fold (median 3011.9 pg/g; range 1051.66-6605.45 pg/g) and 7-fold (median 2263.9; range 994.78-3996.83 pg/g) higher than those in the serum of the same patients (median $299.5 \mathrm{pg} / \mathrm{mL}$; range 87.33-814.35), respectively (Fig. 1A and Table 2). These observations are in line with the fact that DHT is locally produced in the prostate. Moreover, DHEA concentrations in both cancer and benign tissues were 3 -fold higher than those in serum (Fig. 1A and Table 2), while no difference was observed in the tissue/serum ratio between cancer and benign tissue samples. The data, thus, indicate local synthesis or active transport of DHEA and/or DHEA-sulfate into the prostate. The concentrations of A-dione and testosterone in cancer and benign tissues were markedly lower than the total concentrations measured in the serum (Fig. 1A and Table 2). Nevertheless, intratissue testosterone concentrations in both benign and cancer tissue were higher than the free testosterone concentrations in serum in all patients (Fig. 1A). We also compared the cancerous and benign samples of each patients and found out that cancer tissue presented higher DHT concentrations in $71 \%$ of patients (Fig. 1C), with a cancer/benign ratio ranging from 3.4 to 25.8. Altogether, the data revealed a high variability in the intratumoral androgen concentrations ranging from 1052 to $6605 \mathrm{pg} / \mathrm{g}$ for DHT (6-fold difference) and from 100 to $655 \mathrm{pg} / \mathrm{g}$ for testosterone (4-fold difference) (Fig. 1B and Table 2). Since DHT is the most abundant active androgen in the prostate, 

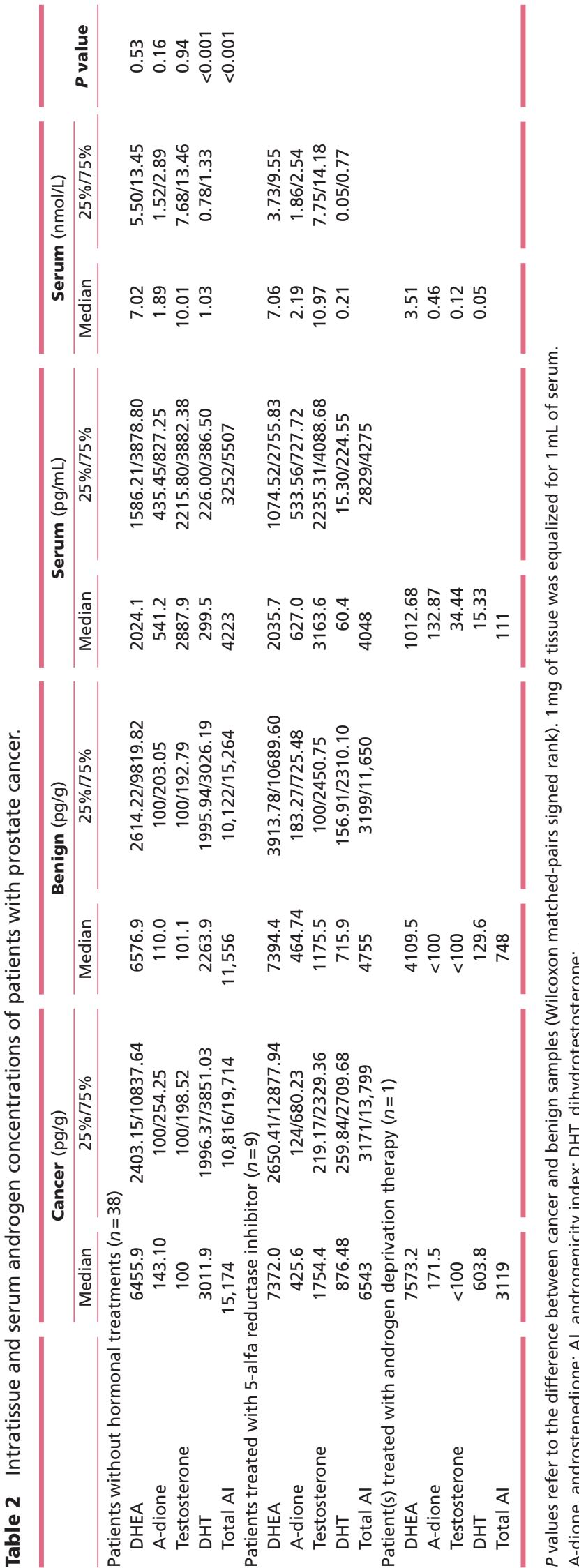

the total androgenicity index followed a similar variability (6-fold difference) as observed for intratumoral DHT (Table 2). Furthermore, the intratumoral concentrations of the low active androgenic precursors, A-dione and DHEA, further indicate broad variability between the patients, ranging from $<100$ to $541 \mathrm{pg} / \mathrm{g}$ for A-dione ( $>5$ fold difference) and from 1242 to $48,375 \mathrm{pg} / \mathrm{g}$ ( 40 -fold difference) for DHEA (Fig. 1B and Table 2). Interestingly, also the tissue/serum ratios of all androgens were highly variable, indicating active and individualized patterns of metabolism and uptake of androgens and their precursors within the prostate, resulting in distinct profiles among the prostate cancer patients (Fig. 1A).

\section{TMPRSS2-ERG positivity is associated with altered intratumoral DHT to testosterone ratio and altered androgen response}

The TMPRSS2-ERG fusion is a frequently found gene fusion in prostate cancer, and thus, its potential association with androgen profiles and changes in the expression of androgen-metabolizing enzymes was of our interest. The patients, who had not received any hormonal therapies $(n=38)$, were classified as TMPRSS2-ERG gene fusion positive (ERG,$+ n=23)$ and TMPRSS2-ERG gene fusion negative (ERG-, $n=15$ ) based on ERG expression measured by RNA-seq (Fig. 2A). The RNA-seq results were validated with IHC (Fig. 2B) in five ERG+ and four ERG- tumors, and a full match with RNA-seq results was obtained. The ERG positivity was also associated with high CRISP3 expression (Fig. 2A and C).

Next, we performed an unsupervised hierarchical clustering of patients based on their cancer/benign ratios for DHEA, A-dione, testosterone and DHT (Fig. 3A). The patients with ERG+ tumors and ERG- tumors were separately clustered, and out of three clusters, two clusters (Cluster 1 and Cluster 2) were enriched with the ERG+ tumors, including $19 \mathrm{ERG}+$ tumors but only four ERG - tumors (Fig. 3A). This result indicates that during the tumor progression, the androgen biosynthesis/ metabolism is differentially altered in the ERG+ and ERGtumors compared to the corresponding benign tissue. Interestingly, three patients diagnosed with biochemical recurrence during the follow-up period, belonged all in Cluster 1 (Fig. 3A and Supplementary Fig. 2). However, the difference between the clusters was not statistically significant using log-rank test. In addition, we compared the concentrations of individual androgens in ERG+ and ERG- cancer specimens and observed that the intratissue levels of testosterone were lower in ERG+ tumors compared 
A

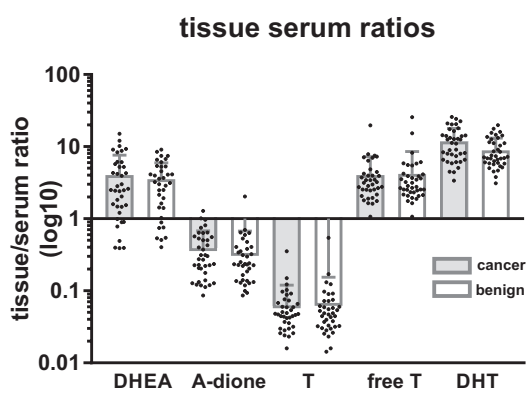

B
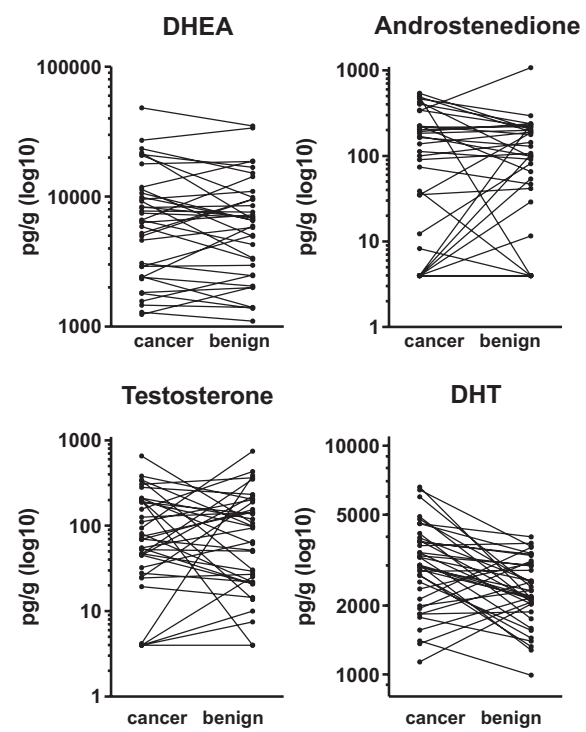

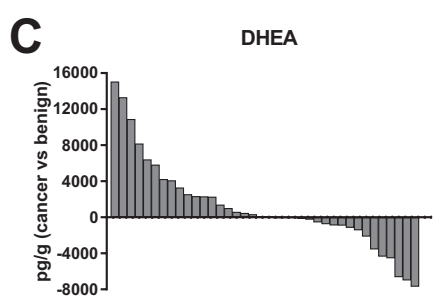

A-dione

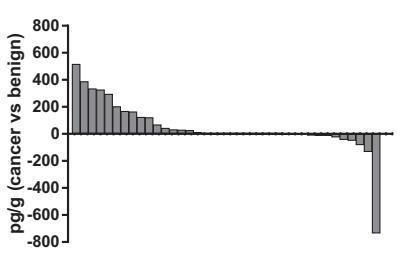

Testosterone

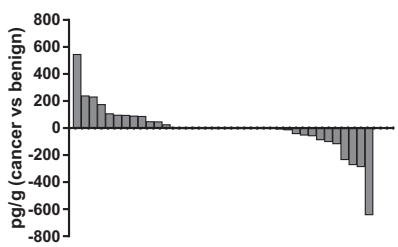

DHT

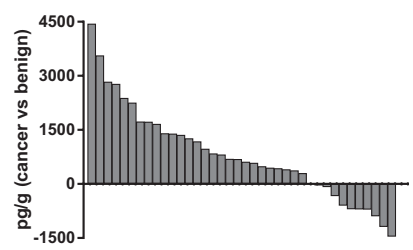

Figure 1

(A) Tissue/serum ratios of DHEA, androstenedione (A-dione), testosterone, free testosterone and dihydrotestosterone (DHT) in cancer (gray) and benign (white) samples obtained from nontreated patients, $n=38$. Error bars represent standard deviation. (B) Intratissue levels of DHEA, A-dione, testosterone and DHT in paired individual cancer and benign tissue samples obtained from non-treated patients $(n=38)$. (C) Waterfall plots demonstrating the differences of intratissue DHEA, A-dione, testosterone and DHT levels between the paired cancer and benign tissue samples $(n=38)$. to ERG - tumors $(P<0.0038$, Fig. 3B). In contrast, the intratissue levels of testosterone in the benign samples of patients were independent of the ERG status of the tumors (Fig. 3B), again suggesting that ERG expression alters the local androgen metabolism. ERG expression was also associated with a trend for higher DHT levels in the tumors, while there was no such trend in benign tissue (Fig. 3B). Also, the levels of DHEA, A-dione and DHT in the benign tissue were similar between the patients with ERG+ and ERG - tumors (Fig. 3B). Accordingly, the DHT/ testosterone ratio in the cancer samples was significantly higher in ERG+ tumors compared to ERG- tumors, while in benign tissue and serum samples, no difference was observed between the ERG+ and ERG- patients (Fig. 3C).

Due to altered DHT/ testosterone ratios, we investigated the gene expression levels of $5 \alpha$-reductase enzymes (SRD5A) converting testosterone to DHT. Interestingly, the gene expression patterns of three $5 \alpha$-reductases enzymes (SRD5A1, SRD5A2 and SRD5A3) were different between the ERG+ and ERG- tumors: $S R D 5 A 3$ expression was higher in ERG+ cancer specimens (Fig. 4A), whereas no significant changes were observed in SRD5A1 and SRD5A2 expression (data not shown). Together with altered DHT/testosterone ratios, the result indicates that the altered profiles of SRD5A enzymes could contribute to testosterone-independent DHT biosynthesis via the alternative pathway. The expression of AR target genes was analyzed using RNA-seq. To our interest, ERG+ and ERG- tumors clustered separately in an unsupervised hierarchical clustering analysis, and $31 \mathrm{AR}$ target genes including ERG were differentially expressed in ERG+ tumors compared to ERG - tumors (Fig. 4B). The differentially expressed genes also included wellcharacterized AR target genes such as NKX3.1, STEAP4 and SPOCK1 (Fig. 4C). Again, no alterations in the expression of these genes were observed in the corresponding benign samples. The expression levels of $A R$ were not changed between cancer and benign samples (Fig. 4B), indicating that the altered androgen response is due to differences in androgen profiles between the ERG+ and ERG- tumors.

\section{Discussion}

In this study, we analyzed androgen concentrations in tissue samples representing both cancerous and 
A
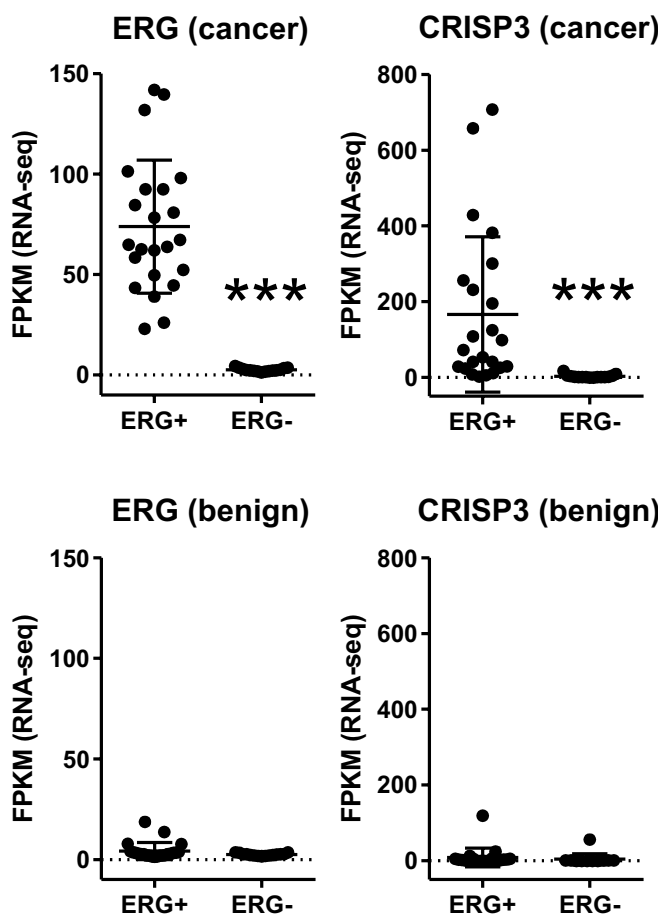

C

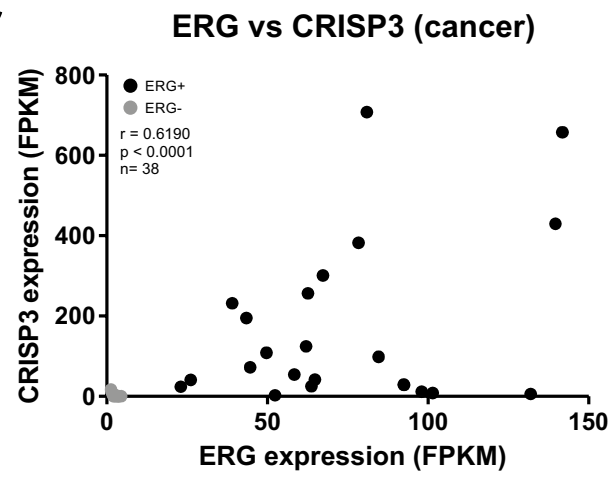

B

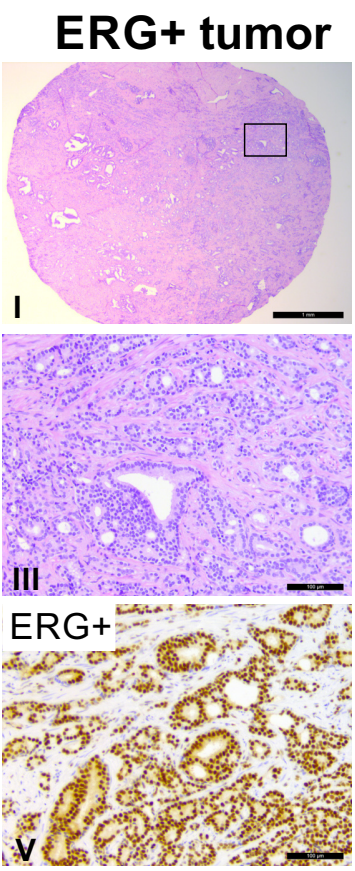

\section{ERG- tumor}

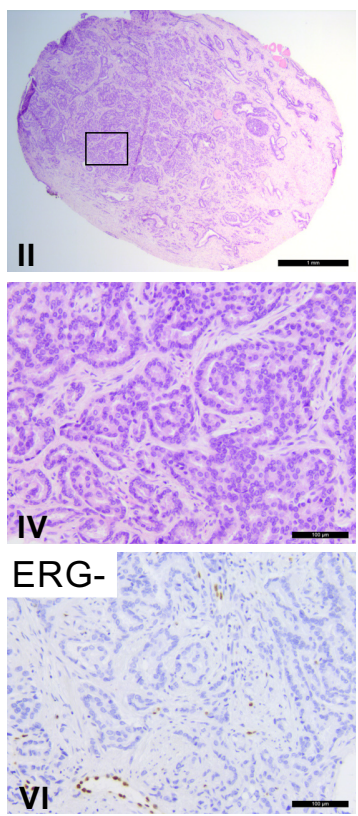

\section{Non-cancerous prostate}

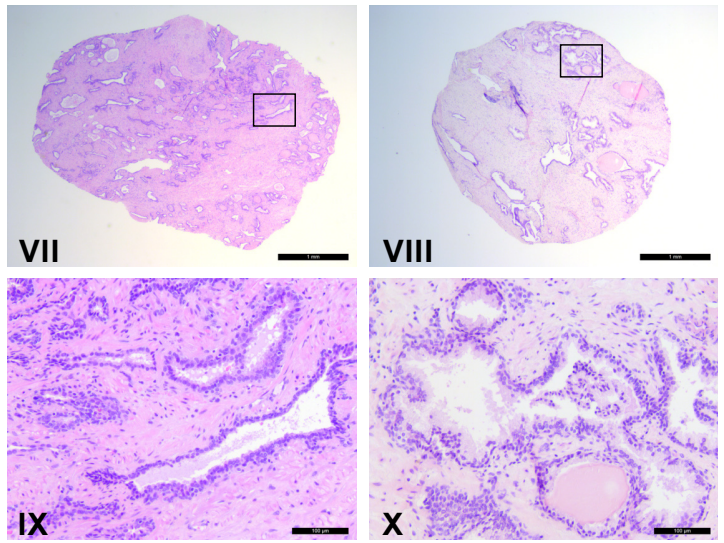

Figure 2

(A) RNA-seq-based mRNA expression of ERG and CRISP3 in ERG+ $(n=23)$ and ERG- $(n=15)$ tumor samples and corresponding benign samples. Adjusted $P$ values: ${ }^{*} * P<0.001$. FPKM $=$ fragments per kilobase of exon per million fragments mapped. Error bars represent standard deviation. (B) Representative pictures of hematoxylin-eosin-stained histological sections of ERG+ (I, III) and ERG- tumors (II, IV), ERG immunohistochemical staining of tumors (ERG+, $n=5$; ERG-, $n=4)$ defined as ERG+ (V) or ERG- $(\mathrm{VI})$ and hematoxylin-eosin stained histological sections of non-cancerous part of prostate from corresponding ERG+ (VII, IX) and ERG - (VIII, X) patients. Scale bars are $1 \mathrm{~mm}$ (I, II, VII, VIII) and 100 Hm (III, IV, V, VI, IX, X). (C) Pearson correlation of ERG and CRISP3 expression measured using RNA-seq in ERG+ $(n=23)$ and ERG- $(n=15)$ tumors.

morphologically benign prostate tissue obtained from primary prostate cancer patients. To improve the comparability of the samples, the cancerous and benign samples were taken from the same individuals. We confirmed that intratissue DHT concentrations were higher in cancer samples compared to the benign tissue of the same prostate. Similar results have been observed previously in some studies (Heracek et al. 2007, Ji et al. 2007), while others have reported higher DHT levels in the benign prostate tissue compared to cancerous samples (Habib et al. 1976, Geller et al. 1978). It should be noted that in the study by Heracek et al. (2007) the benign specimens, collected from patients with benign prostate hyperplasia, represented the benign epithelium of the transition zone of prostate, not the peripheral zone where adenocarcinomas typically occur (McNeal et al. 1988). In contrast, our results provide evidence for the differences in androgen profiles of cancerous and 
A

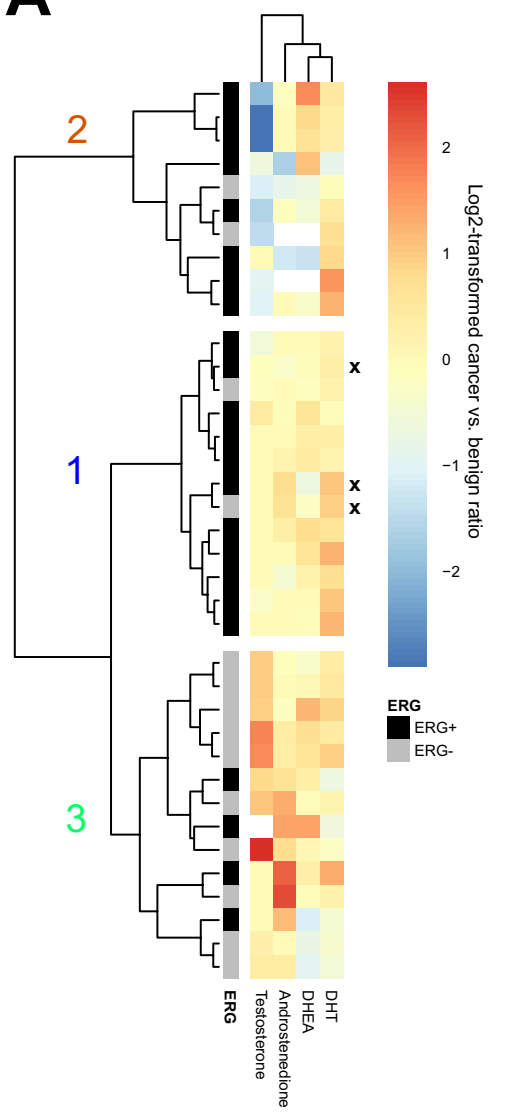

B
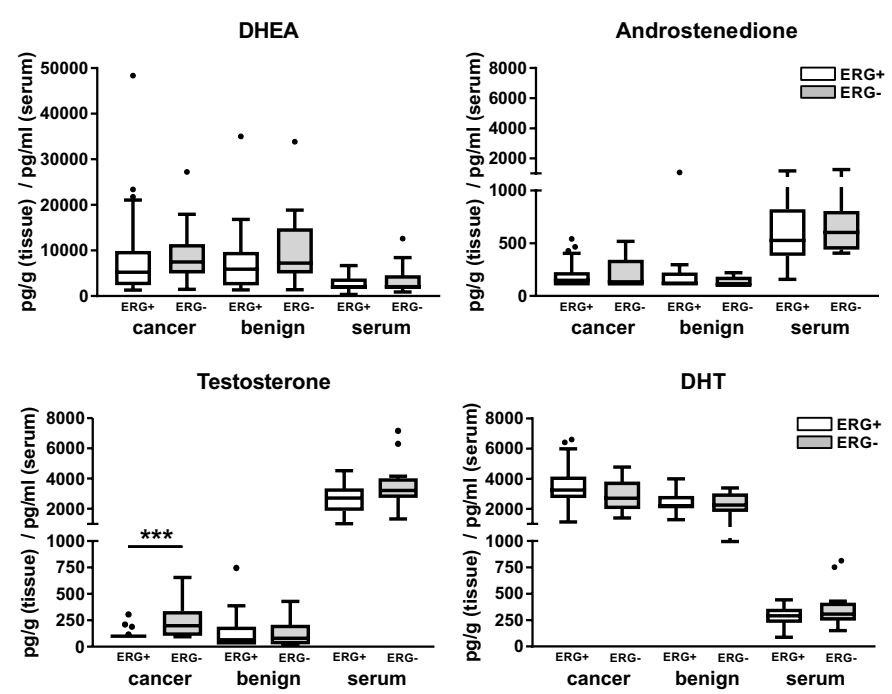

C
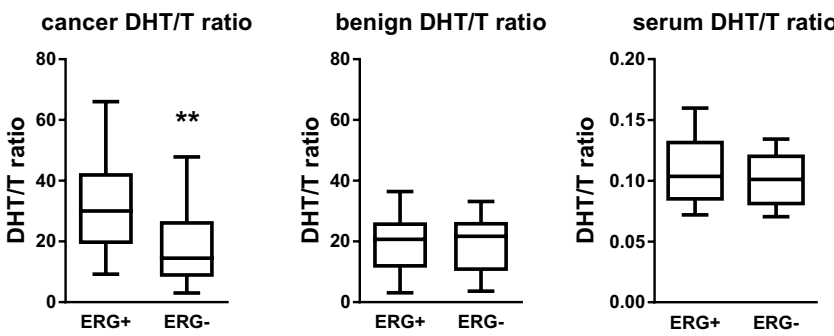

Figure 3

(A) Heatmap of the hierarchical clustering of intratumoral testosterone, androstenedione (A-dione), DHEA and dihydrotestosterone (DHT) concentrations normalized to the corresponding benign tissue concentrations in patients without hormonal treatments $(n=37)$. Cancer samples are hierarchical clustered (Cluster 1, Cluster 2, Cluster 3) and marked with color codes indicating the ERG status (ERG+, ERG-). The patients diagnosed with biochemical recurrence are marked with symbol ' $X$ '. One patient was excluded from the hierarchical clustering due to incomplete androngen profile data. (B) Intratissue and serum levels of DHEA, A-dione, testosterone and DHT in patients with ERG-positive $(n=23)$ and ERG-negative ( $n=15)$ tumors. (C) Intratissue (cancer and benign) and serum DHT/testosterone ratios in ERG-positive (ERG,$+ n=23$ ) and ERG-negative (ERG-, $n=15$ ) patients. Adjusted $P$ values: $* * P<0.01, * * * P<0.001$. All error bars represent standard deviation.

benign prostate, both obtained from the peripheral zone of prostate. This is critical since peripheral and transition zones appear to have different gene expression profiles (van der Heul-Nieuwenhuijsen et al. 2006). Furthermore, many of the previous studies were performed using radioimmunoassays, which suffer from low specificity and cross reactivity problems, while the GC-MS/MS method applied in this study is highly specific with a high accuracy (Nilsson et al. 2015). Thus, the present study demonstrates the individual variability in the intratissue steroid profiles in primary prostate cancer, which in the previous studies may have gone undetected due to their technical limitations. Detection of the altered androgen levels in patients treated with $5 \alpha$-reductase inhibitors (dutasteride and finasteride) or ADT (GnRH antagonist) further confirms the validity of our GC-MS/MS method.
In the present study, we demonstrated that the presence of TMPRSS2-ERG fusion gene in cancer samples is associated with distinct intratumoral androgen profiles. TMPRSS2-ERG is the most frequently found gene fusion in prostate cancer patients, present approximately in half of all patients (Tomlins et al. 2005). The gene fusion between an androgen-regulated TMPRSS2 and oncogenic ERG leads to the overexpression of androgenactivated ERG signaling, which is normally not present in the prostate but has been demonstrated to regulate AR signaling in prostate cancer (Yu et al. 2010, Chen et al. 2013). Furthermore, it has been reported that the AR signaling exposes the prostate cells to TMPRSS2-ERG fusions by inducing proximity of the TMPRSS2 and ERG genomic loci (Lin et al. 2009, Mani et al. 2009). Thus, an amplified androgen-regulated signaling via AR might 
A

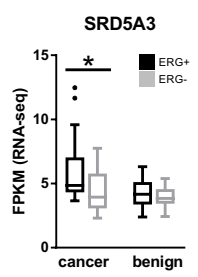

C
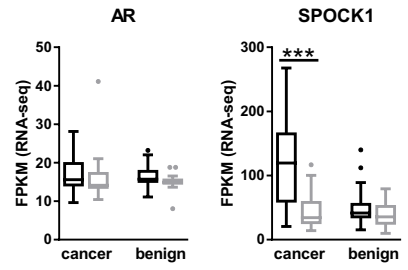
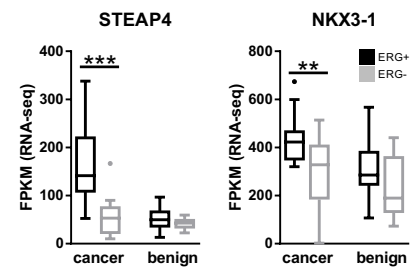

B

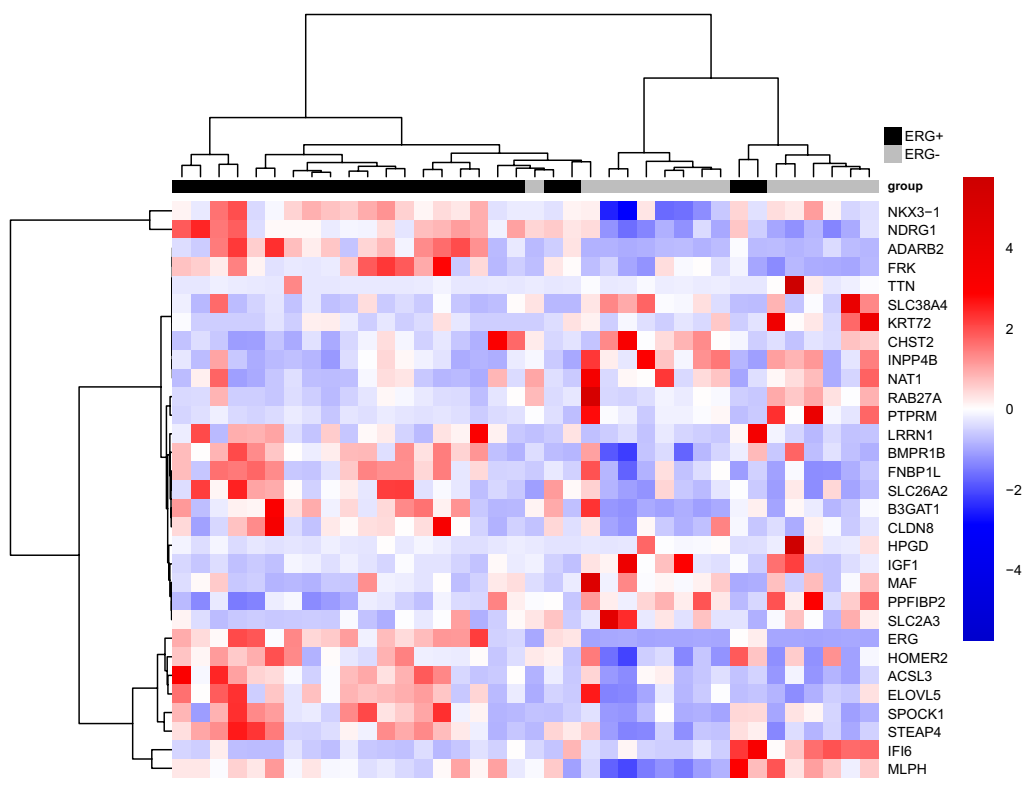

Figure 4

(A) The mRNA expression of SRD5A3 in cancerous and benign samples obtained from patients with ERG+ (boxplots with black lines, $n=23$ ) and ERG - (boxplots with gray lines, $n=15$ ) tumors. (B) Heatmap of unsupervised hierarchical clustering of differentially expressed androgen receptor (AR) target genes in ERG+ $(n=23)$ and ERG$(n=15)$ tumors. (C) The mRNA expression of AR and AR target genes SPOCK1, STEAP4 and NKX3-1 in cancerous and benign samples obtained from patients with ERG+ (boxplots with black lines, $n=23$ ) and ERG - (boxplots with gray lines, $n=15$ ) tumors. Adjusted $P$ values: $* * * P<0.001$, $* * P<0.01, * P<0.05$. FPKM $=$ fragments per kilobase of exon per million fragments mapped. All error bars represent standard deviation.

contribute to the malignant transformation of prostate cells by promoting TMPRSS2-ERG rearrangements.

Several studies have suggested CRISP3, cysteine-rich secretory protein 3 , as a novel biomarker for prostate cancer (Asmann et al. 2002, Bjartell et al. 2007). In line with our results showing a positive correlation between ERG and CRISP3 expression, CRISP3 expression has been demonstrated to be upregulated in prostate carcinomas together with TMPRSS2-ERG fusion gene rearrangements, and the expression of CRISP3 is possibly directly regulated by ERG (Ribeiro et al. 2011). Our findings confirm that CRISP3 expression is strongly associated with ERG expression. The overexpression of CRISP3 has been linked to cell invasion and increased PSA expression in the prostate cancer, suggesting that CRISP3 is involved in the AR signaling (Pathak et al. 2016).

Our present study provides further evidence for the association between ERG and androgen signaling in prostate cancer. The unsupervised clustering using cancer/ benign ratios of intratissue androgen levels demonstrated that the androgen metabolism and/or transport is differentially altered in ERG+ tumors. We observed that low intratumoral and serum levels of testosterone are associated with high $E R G$ expression in cancer samples, while no such association was found in benign prostate samples from the same patients. Furthermore, intratissue DHT/ testosterone ratios were significantly higher in ERG+ tumors, accompanied by a tendency toward a higher intratumoral DHT levels. A recent study suggested that high pre-diagnostic levels of free testosterone in serum are associated with an increased risk of developing TMPRSS2ERG-positive prostate cancer (Graff et al. 2016). However, our results associated low total testosterone levels in serum with TMPRSS2-ERG-positive tumors. Moreover, ERG has been demonstrated to directly inhibit the $A R$ expression and disrupt AR signaling in prostate cancer (Yu et al. 2010), while in the present study, we did not find any differences in the AR expression between the cancer or benign samples or ERG+ and ERG- tumors. However, the biological significance of the altered intratumoral androgen profile in ERG+ tumors was evidenced by a distinct AR target gene signature without differences in $A R$ mRNA expression. Numerous AR target genes, including NKX3.1, SPOCK1 and STEAP4, were upregulated in ERG+ 
tumors, indicating an enhanced AR signaling, possibly contributing to the prevalence of TMPRSS2-ERG fusions as suggested by others (Lin et al. 2009, Mani et al. 2009).

ERG+ and ERG- patients mainly distributed to separate clusters when the cancer/benign ratios of intratissue androgen levels for each patient were analyzed. The clustering, together with the difference in intratumoral DHT/testosterone ratio between the ERG+ and ERG- cancer specimens, suggest that intratissue androgen metabolism is altered by ERG expression. One explanation for the increased DHT/testosterone ratios in ERG+ tumors is that the intratumoral testosterone is converted more efficiently to DHT by $5 \alpha$-reductases. Unlike SRD5A1 and SRD5A2, the mRNA expression level of $S R D 5 A 3$ was significantly upregulated in the ERG+ tumors compared to ERG - tumors. The role of SRD5A3 in prostate cancer androgen metabolism is not well established, but the enzyme has been demonstrated to be overexpressed in CRPC cells (Uemura et al. 2008).

DHT can be produced in prostate cancer cells via an alternative pathway that does not depend on the production of testosterone (Chang et al. 2011). The pathway involves $5 \alpha$-reductase activity, converting A-dione to androstanedione (5 $\alpha$-dione), which is subsequently converted to DHT by HSD17B activity (Chang et al. 2011). One of the enzymes contributing to this HSD17B activity is AKR1C3 that is overexpressed in CRPC samples (Fankhauser et al. 2014). Also, 5 $\alpha$-dione treatment induces DHT production, and consequently, AR activation in VCaP cells, while both ERG or AKR1C3 knockdown significantly reduce DHT production, supporting the findings that ERG can directly regulate the AKR1C3 expression (Powell et al. 2015). Our data, demonstrating the increased DHT/testosterone ratios in ERG+ tumors, would suggest the activation of the alternative pathway in which DHT is synthetized from $5 \alpha$-dione, bypassing testosterone production. However, we found no differences in the AKR1C3 mRNA expression (data not shown) between the ERG+ and ERG- tumors. Whether steroidogenic enzyme expression is altered by a direct activation of ERG in TMPRSS2-ERG positive tumors remains to be studied.

Taken together, we have shown that the patients diagnosed with TMPRSS2-ERG-positive prostate cancer have distinct intratissue androgen profiles compared to TMPRSS2-ERG-negative patients. Higher DHT/testosterone ratios in TMPRSS2-ERG-positive prostate tumors suggest an activation of the alternative pathway leading to testosterone-independent DHT production. The TMPRSS2-ERG positivity was also associated with an induced AR target gene expression. Thus, the patients diagnosed with TMPRSS2-ERG-positive prostate cancer may benefit from novel drugs inhibiting testosteroneindependent androgen biosynthesis.

\section{Supplementary data}

This is linked to the online version of the paper at https://doi.org/10.1530/ ERC-18-0148.

\section{Declaration of interest}

The authors declare that there is no conflict of interest that could be perceived as prejudicing the impartiality of the research reported.

\section{Funding}

This work was supported by the Finnish Cultural Foundation (M K), Drug Research Doctoral Program (M K), Cancer Society of Finland (M K, P T and $M P$ ), Sigrid Juselius Foundation ( $L L E$ ), the Academy of Finland ( $L E E$, grant number 296801) and Orion Pharma (M P).

\section{Acknowledgements}

The authors are thankful to the personnel working at Turku University Hospital and Auria Biobank, and all collaborators involved in Turku Prostate Cancer Consortium (TPCC). Pauliina Toivonen and Saana Viertomanner are acknowledged for technical assistance in sample collection and preparation, Sanni Tuominen and Elina Komsi for help with RNA/DNA extraction, and Sinikka Collanus for help with immunohistochemistry. They especially want to thank all patients who participated in this study.

\section{References}

Asmann YW, Kosari F, Wang K, Cheville JC \& Vasmatzis G 2002 Identification of differentially expressed genes in normal and malignant prostate by electronic profiling of expressed sequence tags. Cancer Research 62 3308-3314.

Bjartell AS, Al-Ahmadie H, Serio AM, Eastham JA, Eggener SE, Fine SW, Udby L, Gerald WL, Vickers AJ, Lilja H, et al. 2007 Association of cysteine-rich secretory protein 3 and beta-microseminoprotein with outcome after radical prostatectomy. Clinical Cancer Research $\mathbf{1 3}$ 4130-4138. (https://doi.org/10.1158/1078-0432.CCR-06-3031)

Cai C \& Balk SP 2011 Intratumoral androgen biosynthesis in prostate cancer pathogenesis and response to therapy. Endocrine-Related Cancer 18 R175-R182. (https://doi.org/10.1530/ERC-10-0339)

Chang K-H, Li R, Papari-Zareei M, Watumull L, Zhao YD, Auchus RJ \& Sharifi N 2011 Dihydrotestosterone synthesis bypasses testosterone to drive castration-resistant prostate cancer. PNAS 108 13728-13733. (https://doi.org/10.1073/pnas.1107898108)

Chen Y, Chi P, Rockowitz S, Iaquinta PJ, Shamu T, Shukla S, Gao D, Sirota I, Carver BS, Wongvipat J, et al. 2013 ETS factors reprogram the androgen receptor cistrome and prime prostate tumorigenesis in response to PTEN loss. Nature Medicine 19 1023-1029. (https://doi. org/10.1038/nm.3216)

de Bono JS, Logothetis CJ, Molina A, Fizazi K, North S, Chu L, Chi KN, Jones RJ, Goodman OB, Saad F, et al. 2011 Abiraterone and increased survival in metastatic prostate cancer. New England Journal of Medicine 364 1995-2005. (https://doi.org/10.1056/NEJMoa1014618)
C) 2018 Society for Endocrinology Published by Bioscientifica Ltd. Printed in Great Britain 
Fankhauser M, Tan Y, Macintyre G, Haviv I, Hong MKH, Nguyen A, Pedersen JS, Costello AJ, Hovens CM \& Corcoran NM 2014 Canonical androstenedione reduction is the predominant source of signaling androgens in hormone-refractory prostate cancer. Clinical Cancer Research 20 5547-5557. (https://doi.org/10.1158/1078-0432. CCR-13-3483)

Geller J, Albert J, de la Vega D, Loza D \& Stoeltzing W 1978 Dihydrotestosterone concentration in prostate cancer tissue as a predictor of tumor differentiation and hormonal dependency. Cancer Research 38 4349-4352.

Graff RE, Meisner A, Ahearn TU, Fiorentino M, Loda M, Giovannucci EL, Mucci LA \& Pettersson A 2016 Pre-diagnostic circulating sex hormone levels and risk of prostate cancer by ERG tumour protein expression. British Journal of Cancer 114 939-944. (https://doi. org/10.1038/bjc.2016.61)

Habib FK, Lee IR, Stitch SR \& Smith PH 1976 Androgen levels in the plasma and prostatic tissues of patients with benign hypertrophy and carcinoma of the prostate. Journal of Endocrinology 71 99-107. (https://doi.org/10.1677/joe.0.0710099)

Heracek J, Richard H, Martin H, Luboslav S, Jana S, Jitka K, Vaclav E, Michael U \& Vaclav M 2007 Tissue and serum levels of principal androgens in benign prostatic hyperplasia and prostate cancer. Steroids 72 375-380. (https://doi.org/10.1016/j.steroids.2007.01.004)

Huhtinen K, Saloniemi-Heinonen T, Keski-Rahkonen P, Desai R, Laajala D, Stahle M, Häkkinen MR, Awosanya M, Suvitie P, Kujari H, et al. 2014 Intra-tissue steroid profiling indicates differential progesterone and testosterone metabolism in the endometrium and endometriosis lesions. Journal of Clinical Endocrinology and Metabolism 99 E2188-E2197. (https://doi.org/10.1210/jc.2014-1913)

Ji Q, Chang L, Stanczyk FZ, Ookhtens M, Sherrod A \& Stolz A 2007 Impaired dihydrotestosterone catabolism in human prostate cancer: Critical role of AKR1C2 as a pre-receptor regulator of androgen receptor signaling. Cancer Research 67 1361-1369. (https://doi org/10.1158/0008-5472.CAN-06-1593)

Keski-Rahkonen P, Huhtinen K, Poutanen M \& Auriola S 2011 Fast and sensitive liquid chromatography-mass spectrometry assay for seven androgenic and progestagenic steroids in human serum. Journal of Steroid Biochemistry and Molecular Biology 127 396-404. (https://doi. org/10.1016/j.jsbmb.2011.06.006)

Knuuttila M, Yatkin E, Kallio J, Savolainen S, Laajala TD, Aittokallio T, Oksala R, Häkkinen M, Keski-Rahkonen P, Auriola S, et al. 2014 Castration induces up-regulation of intratumoral androgen biosynthesis and androgen receptor expression in an orthotopic VCaP human prostate cancer xenograft model. American Journal of Pathology 184 2163-2173. (https://doi.org/10.1016/j. ajpath.2014.04.010)

Lin C, Yang L, Tanasa B, Hutt K, Ju BG, Ohgi K, Zhang J, Rose DW, Fu XD, Glass CK, et al. 2009 Nuclear receptor-induced chromosomal proximity and DNA breaks underlie specific translocations in cancer. Cell 139 1069-1083. (https://doi.org/10.1016/j.cell.2009.11.030)

Mani R-S, Tomlins SA, Callahan K, Ghosh A, Nyati MK, Varambally S, Palanisamy N \& Chinnaiyan AM 2009 Induced chromosomal proximity and gene fusions in prostate cancer. Science $\mathbf{3 2 6} 1230$. (https://doi.org/10.1126/science.1178124)

McNeal JE, Redwine EA, Freiha FS \& Stamey TA 1988 Zonal distribution of prostatic adenocarcinoma. Correlation with histologic pattern and direction of spread. American Journal of Surgical Pathology 12 897-906.

Mostaghel EA 2014 Beyond T and DHT - novel steroid derivatives capable of wild type androgen receptor activation. International Journal of Biological Sciences 10 602-613. (https://doi.org/10.7150/ ijbs.8844)

Mostaghel EA \& Nelson PS 2008 Intracrine androgen metabolism in prostate cancer progression: mechanisms of castration resistance and therapeutic implications. Best Practice and Research: Clinical
Endocrinology and Metabolism 22 243-258. (https://doi.org/10.1016/j. beem.2008.01.003)

Nicorici D, Satalan M, Edgren H, Kangaspeska S, Murumagi A, Kallioniemi O, Virtanen S \& Kilkku O 2014 FusionCatcher - a tool for finding somatic fusion genes in paired-end RNA-sequencing data. bioRxiv. (https://doi.org/10.1101/011650)

Nilsson ME, Vandenput L, Tivesten Å, Norlén A-K, Lagerquist MK, Windahl SH, Börjesson AE, Farman HH, Poutanen M, Benrick A, et al. 2015 Measurement of a comprehensive sex steroid profile in rodent serum by high-sensitive gas chromatography-tandem mass spectrometry. Endocrinology 156 en.2014-en.1890. (https://doi. org/10.1210/en.2014-1890)

Page ST, Lin DW, Mostaghel E a, Hess DL, True LD, Amory JK, Nelson PS, Matsumoto AM \& Bremner WJ 2006 Persistent intraprostatic androgen concentrations after medical castration in healthy men. Journal of Clinical Endocrinology and Metabolism 91 3850-3856. (https://doi.org/10.1210/jc.2006-0968)

Pathak BR, Breed AA, Apte S, Acharya K \& Mahale SD 2016 Cysteinerich secretory protein 3 plays a role in prostate cancer cell invasion and affects expression of PSA and ANXA1. Molecular and Cellular Biochemistry 411 11-21. (https://doi.org/10.1007/s11010-015-2564-2)

Penning TM 2014 Androgen biosynthesis in castration-resistant prostate cancer. Endocrine-Related Cancer 21 T67-T78. (https://doi. org/10.1530/ERC-14-0109)

Powell K, Semaan L, Conley-LaComb MK, Asangani I, Wu Y-M, Ginsburg KB, Williams J, Squire JA, Maddipati KR, Cher ML, et al. 2015 ERG/AKR1C3/AR constitutes a feed-forward loop for AR signaling in prostate cancer cells. Clinical Cancer Research 21 2569-2579. (https://doi.org/10.1158/1078-0432.CCR-14-2352)

Quanson JL, Stander MA, Pretorius E, Jenkinson C, Taylor AE \& Storbeck KH 2016 High-throughput analysis of 19 endogenous androgenic steroids by ultra-performance convergence chromatography tandem mass spectrometry. Journal of Chromatography B: Analytical Technologies in the Biomedical and Life Sciences 1031 131138. (https://doi.org/10.1016/j.jchromb.2016.07.024)

Ribeiro FR, Paulo P, Costa VL, Barros-Silva JD, Ramalho-Carvalho J, Jerónimo C, Henrique R, Lind GE, Skotheim RI, Lothe RA, et al. 2011 Cysteine-Rich secretory protein-3 (CRISP3) is strongly Up-Regulated in prostate carcinomas with the TMPRSS2-ERG fusion gene. PLoS ONE 6 e22317. (https://doi.org/10.1371/journal.pone.0022317)

Ritchie ME, Phipson B, Wu D, Hu Y, Law CW, Shi W \& Smyth GK 2015 Limma powers differential expression analyses for RNA-sequencing and microarray studies. Nucleic Acids Research 43 e47. (https://doi. org/10.1093/nar/gkv007)

Robinson MD, McCarthy DJ \& Smyth GK 2010 edgeR: a Bioconductor package for differential expression analysis of digital gene expression data. Bioinformatics 26 139-140. (https://doi.org/10.1093/ bioinformatics/btp616)

Ryan CJ, Smith MR, De Bono JS, Molina A, Logothetis CJ, De Souza P, Fizazi K, Mainwaring P, Piulats JM, Ng S, et al. 2013 Abiraterone in metastatic prostate cancer without previous chemotherapy. New England Journal of Medicine 368 138-148. (https://doi.org/10.1056/ NEJMoa1209096)

Seyednasrollah F, Rantanen K, Jaakkola P \& Elo LL 2015 ROTS: reproducible RNA-seq biomarker detector-prognostic markers for clear cell renal cell cancer. Nucleic Acids Research 44 e1. (https://doi. org/10.1093/nar/gkv806)

Tamae D, Byrns M, Marck B, Mostaghel EA, Nelson PS, Lange P, Lin D, Taplin ME, Balk S, Ellis W, et al. 2013 Development, validation and application of a stable isotope dilution liquid chromatography electrospray ionization/selected reaction monitoring/mass spectrometry (SID-LC/ESI/SRM/MS) method for quantification of keto-androgens in human serum. Journal of Steroid Biochemistry and Molecular Biology 138 281-289. (https://doi.org/10.1016/j. jsbmb.2013.06.014)
(2) 2018 Society for Endocrinology Published by Bioscientifica Ltd. Printed in Great Britain 
Titus MA, Schell MJ, Lih FB, Tomer KB \& Mohler JL 2005 Testosterone and dihydrotestosterone tissue levels in recurrent prostate cancer recurrent prostate cancer. Clinical Cancer Research $114653-4657$. (https://doi.org/10.1158/1078-0432.CCR-05-0525)

Tomlins SA, Rhodes DR, Perner S, Dhanasekaran SM, Mehra R, Sun X-W, Varambally S, Cao X, Tchinda J, Kuefer R, et al. 2005 Recurrent fusion of TMPRSS2 and ETS transcription factor genes in prostate cancer. Science 310 644-648. (https://doi.org/10.1126/ science.1117679)

Uemura M, Tamura K, Chung S, Honma S, Okuyama A, Nakamura Y \& Nakagawa H 2008 Novel $5 \alpha$-steroid reductase (SRD5A3, type-3) is overexpressed in hormone-refractory prostate cancer. Cancer Science 99 81-86. (https://doi.org/10.1111/j.1349-7006.2007.00656.x)

van der Heul-Nieuwenhuijsen L, Hendriksen PJM, van der Kwast TH \& Jenster G 2006 Gene expression profiling of the human prostate zones. BJU International 98 886-897. (https://doi. org/10.1111/j.1464-410X.2006.06427.x)

Van Der Sluis TM, Vis AN, Van Moorselaar RJA, Bui HN, Blankenstein MA, Meuleman EJH \& Heijboer AC 2012 Intraprostatic testosterone and dihydrotestosterone. Part I: concentrations and methods of determination in men with benign prostatic hyperplasia and prostate cancer. BJU International 109 176-182. (https://doi org/10.1111/j.1464-410X.2011.10651.x)

Vermeulen A, Verdonck L \& Kaufman JM 1999 A critical evaluation of simple methods for the estimation of free testosterone in serum. Journal of Clinical Endocrinology and Metabolism 84 3666-3672. (https://doi.org/10.1210/jcem.84.10.6079)

Wilson JD 2001 The role of 5alpha-reduction in steroid hormone physiology. Reproduction, Fertility, and Development 13 673-678.

Yu J, Yu J, Mani RS, Cao Q, Brenner CJ, Cao X, Wang X, Wu L, Li J, $\mathrm{Hu} \mathrm{M}$, et al. 2010 An integrated network of androgen receptor, polycomb, and TMPRSS2-ERG gene fusions in prostate cancer progression. Cancer Cell 17 443-454. (https://doi.org/10.1016/j. ccr.2010.03.018)

Zhang A, Zhang J, Plymate S \& Mostaghel EA 2016 Classical and nonclassical roles for pre-receptor control of DHT metabolism in prostate cancer progression. Hormones and Cancer 7 104-113. (https://doi. org/10.1007/s12672-016-0250-9)

Received in final form 15 May 2018

Accepted 17 May 2018

Accepted Preprint published online 17 May 2018
(C) 2018 Society for Endocrinology Published by Bioscientifica Ltd. Printed in Great Britain 